\title{
MEASUREMENT AND OPTIMISATION OF THE PS-SPS TRANSFER LINE OPTICS
}

\author{
G. Arduini, M. Giovannozzi, K. Hanke* , D. Manglunki, M. Martini, G. Métral \\ CERN, 1211 Geneva 23, Switzerland
}

\section{Abstract}

The beam optics of the PS-SPS transfer line at CERN has been studied and optimised for a variety of beams. Betatron and dispersion matching has been performed for the fixedtarget proton and ion beams, as well as for the future LHC proton beam. The techniques applied to the measurement of the optical parameters in the transfer line are discussed and experimental results are presented.

\section{INTRODUCTION}

The TT2/TT10 beam line is used to transfer from PS to SPS protons and lead ions for fixed-target physics, protons to simulate the future LHC-type beam and positrons for LEP. In recent years, there has been a renewed interest in the optics of this transfer line for two main reasons.

Firstly, the tight emittance budget for the LHC beam. The maximum allowed emittance blow-up in the transfer from PS extraction to SPS extraction is $17 \%$ and only a small fraction of this is assigned to mismatch at injection. Since this beam has a small emittance and a large momentum spread [1], dispersion mismatch is a major concern.

Secondly, high-intensity proton and lead ion beams are required by the physics community, therefore minimum emittance blow-up is needed to minimise beam losses and thus increase the beam intensity delivered to the experiments.

For these reasons, a measurement campaign was undertaken during the 1998 physics run to define and improve the model of the TT2/TT10 transfer line $[2,3]$ and to measure the optical parameters $\alpha, \beta$, the dispersion $D$ and its derivative $D^{\prime}$ so that improved optics could be computed and tested in operation.

\section{DEFINITION AND MEASUREMENT OF INJECTION BLOW-UP FACTORS}

The Twiss parameters at any point in a transfer line depend not only on the settings of the magnets but also on the values of $\alpha$ and $\beta$ at the beginning of the line. Therefore their knowledge is mandatory to carry out optics calculations.

The initial values of $\alpha$ and $\beta$ can be obtained by measuring the optical parameters at a given location and then by back-propagating them to the entry point of the line. The optical parameters can be obtained by measuring the beam profile at three different locations in the line, using e.g. secondary emission monitors (SEMs) (three of them

\footnotetext{
*Email: Klaus.Hanke@cern.ch
}

are installed in TT2 and three in TT10) or optical transition radiation (OTR) monitors (three of such monitors are installed in TT10). Under the hypothesis that the transfer matrices between the three monitors are known as well as the value of $D$ at the three monitor locations ${ }^{1}$, the beam emittance, $\alpha$ and $\beta$ can be computed [5].

To evaluate the blow-up due to the mismatch of the Twiss parameters $\alpha, \beta$ at injection the geometrical blow-up $G_{b}$ and the blow-up after filamentation $H$ are defined [5]. $G_{b}$ is the ratio between the area of the circle having the major axis of the mismatched ellipse as diameter and the area of the circle representing the matched beam. $H$ provides the emittance blow-up once the dilution due filamentation is taken into account, namely:

$$
\begin{gathered}
G_{b}=H+\sqrt{H^{2}-1} \\
H=\frac{1}{2}\left[\frac{\beta_{0}}{\beta_{m}}+\left(\alpha_{0}-\alpha_{m} \frac{\beta_{0}}{\beta_{m}}\right)^{2} \frac{\beta_{m}}{\beta_{0}}+\frac{\beta_{m}}{\beta_{0}}\right] .
\end{gathered}
$$

In Eq. (2), $\alpha_{m}, \beta_{m}$ stand for the measured Twiss parameters while $\alpha_{0}, \beta_{0}$ are the nominal Twiss parameters. The blow-up after filamentation, which is the relevant quantity, is always smaller than the geometrical blow-up.

The measurement of the dispersion is performed by varying the momentum of the beam extracted from PS in steps and by recording the transverse displacement at each available beam position monitor (BPM) in TT2/TT10 and in the SPS first-turn (see Fig. 1) considered as a continuation of the transfer line. The momentum offset $\Delta p$ with

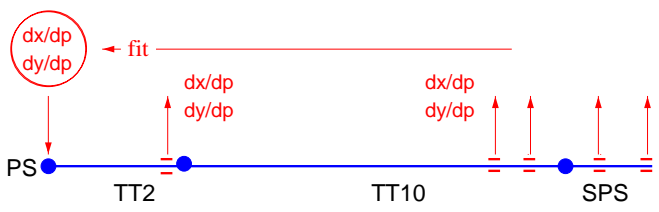

Figure 1: Schematic representation of the dispersion measurement

respect to the reference momentum $p$ is calculated by measuring the change in radial position $\Delta R$ of the beam in its first turn according to

$$
\frac{\Delta p}{p}=\frac{\Delta R}{\alpha_{p}},
$$

provided the momentum compaction factor $\alpha_{p}$ of the SPS machine is known. The beam displacement measurements

\footnotetext{
${ }^{1}$ This last condition can be dropped, provided more profiles are measured, see [4].
} 
as a function of the momentum offset are fitted to a straight line for each BPM and the slope $d x / d p$ is obtained. A three-parameter $\left((d x / d p)_{m},\left(d x^{\prime} / d p\right)_{m}, \delta\right)$ least-squares fit of the calculated $(d x / d p)_{i}$ to the function

$$
C_{i}\left(\frac{d x}{d p}\right)_{m}+S_{i}\left(\frac{d x^{\prime}}{d p}\right)_{m}+\xi_{i} \delta
$$

is performed in the horizontal plane while in the vertical plane $\delta=1$ is assumed. $C_{i}, S_{i}, \xi_{i}$ are the elements of the $3 \times 3$ transfer matrix [5] from the measurement point $m$ to the $i$-th beam position monitor and $\delta$ is a calibration factor to correct for possible scaling errors in the measurement of the momentum offset. The value of the parameter $\delta$ obtained from the fit of the data in the horizontal plane is used to correct the momentum offsets considered for the data relative to the vertical plane. As a result of the fit,

$$
D_{m}^{f i t}=\frac{(d x / d p)_{m}^{f i t}}{\delta^{f i t}} \quad D_{m}^{\prime} f i t=\frac{\left(d x^{\prime} / d p\right)_{m}^{f i t}}{\delta^{f i t}}
$$

at any point in the line if the transfer matrix from the measurement point to any BPM is known. The presented method takes advantage of the large number of BPMs in the SPS ring (108 per plane) and provides an immediate picture of the mismatch with respect to the dispersion in the ring but it requires beams with small emittance and small momentum spread.

In the presence of dispersion mismatch, the beam will blow-up. These effects can be quantified by means of the geometrical blow-up $G_{d}$ and the blow-up after filamentation $J[6]$

$$
\begin{gathered}
G_{d}=2 J-1 \\
J=1+\frac{\Delta D^{2}+\left(\Delta D^{\prime} \beta_{0}+\Delta D \alpha_{0}\right)^{2}}{2 \varepsilon_{\text {r.m.s. }} \beta_{0}}\left(\frac{\Delta p}{p}\right)_{\text {r.m.s. }}^{2}
\end{gathered}
$$

where $(\Delta p / p)_{r . m . s .}$ and $\varepsilon_{\text {r.m.s. }}$ are the r.m.s. momentum spread and r.m.s. emittance, $\alpha_{0}, \beta_{0}$ are the nominal Twiss parameters and $\Delta D\left(\Delta D^{\prime}\right)$ is the difference between theoretical and measured dispersion (dispersion derivative).

We stress that to derive the expression for $G_{b}, H, G_{d}, J$ one assumes to have filled ellipses and no linear coupling.

\section{EXPERIMENTAL RESULTS}

For the different beam types ( $26 \mathrm{GeV} / \mathrm{c}$ fast extracted proton beam, $14 \mathrm{GeV} / \mathrm{c}$ continuous transfer proton beam and fast extracted lead ion beam) the same measurement procedure has been applied. Firstly $D$ has been measured in TT2/TT10, then the Twiss parameters have been deduced using the SEMs in TT2 and TT10. Finally, the optical parameters at the entry point of the transfer line have been obtained by tracking back the values at the SEM locations. The knowledge of the model and of the initial optical parameters has allowed the computation of new optics for the transfer line with reduced blow-up.

\subsection{The $26 \mathrm{GeV} / \mathrm{c}$ fast extracted beam}

The $26 \mathrm{GeV} / \mathrm{c}$ fast extracted proton beam is the so-called LHC beam. The detailed beam parameters can be found in [7]. The measured values of $D_{H}$ in TT2/TT10 and

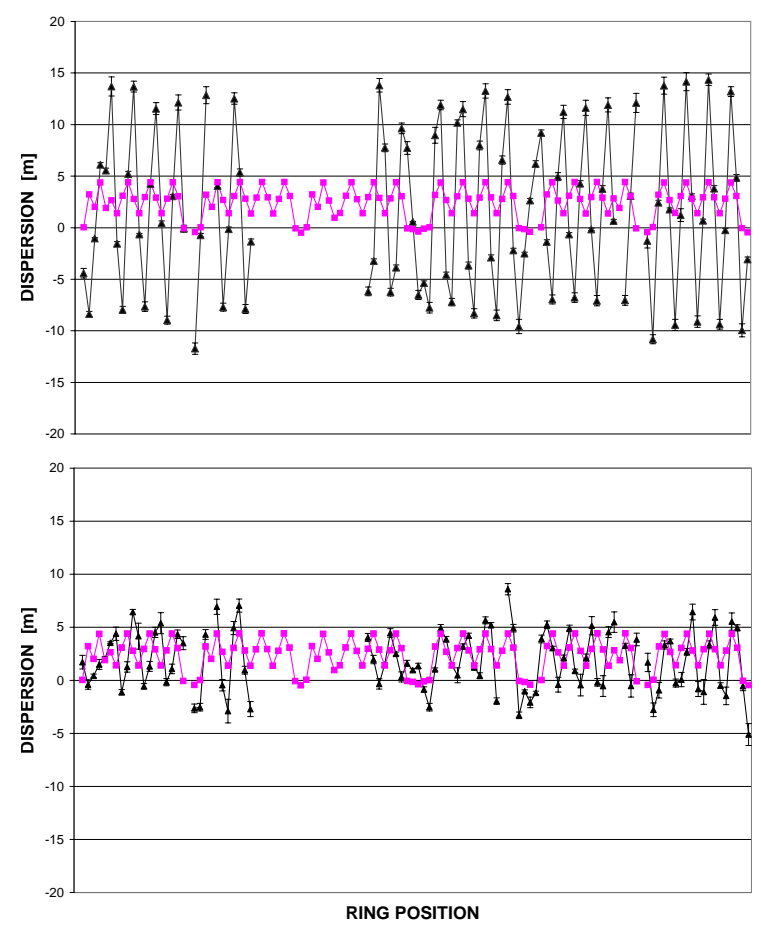

Figure 2: Measured (darker triangles) and theoretical (squares) horizontal dispersion in the SPS ring before (upper) and after (lower) matching the injection line

the SPS for the initial optics (upper) and the matched one (lower) are shown in Fig. 2. The overall reduction of $D_{H}$ is clearly visible. The values of the mismatch factors after filamentation for the old and new optics are listed in Table 1. The improvement is remarkable, even though the blow-up

\begin{tabular}{|c|c||c|}
\hline & Old optics & New optics \\
\hline$H$ (H-plane) & 1.1 & 1.0 \\
$H$ (V-plane) & 1.3 & 1.0 \\
$J$ (H-plane) & 11.6 & 1.7 \\
$J$ (V-plane) & 1.0 & 1.0 \\
\hline
\end{tabular}

Table 1: Measured filamented betatron and dispersion blow-up factors for old and new optics

due to $D_{H}$ is far from the nominal value imposed by the real LHC emittance budget. Further studies are needed to improve this situation.

\subsection{The $14 \mathrm{GeV/c}$ continuous transfer beam}

The control of the mismatch for the high intensity (about $3 \times 10^{13} \mathrm{ppp}$ ) $14 \mathrm{GeV} / \mathrm{c}$ proton beam for fixed target physics is mandatory in order to reduce beam losses and thus increase the intensity delivered to the targets. For this beam the TT2/TT10 line is set-up with an emittance exchange 
section to match the beam envelope with the physical aperture of the SPS.

A new optics tested in operation (see [8]) allowed the reduction of the injection losses by a factor two and the increase of the transmission efficiency by $2 \%$, reaching a value of $92 \%$. Furthermore, the improved knowledge of the optical parameters allowed the elimination of a longlasting discrepancy between the emittance values measured in TT2 and TT10. In Fig. 3 the beam emittance measured in TT2 and TT10 as a function of time during the physics run is shown. The better agreement is correlated with the use of the measured optical parameter in the emittance measurement program.

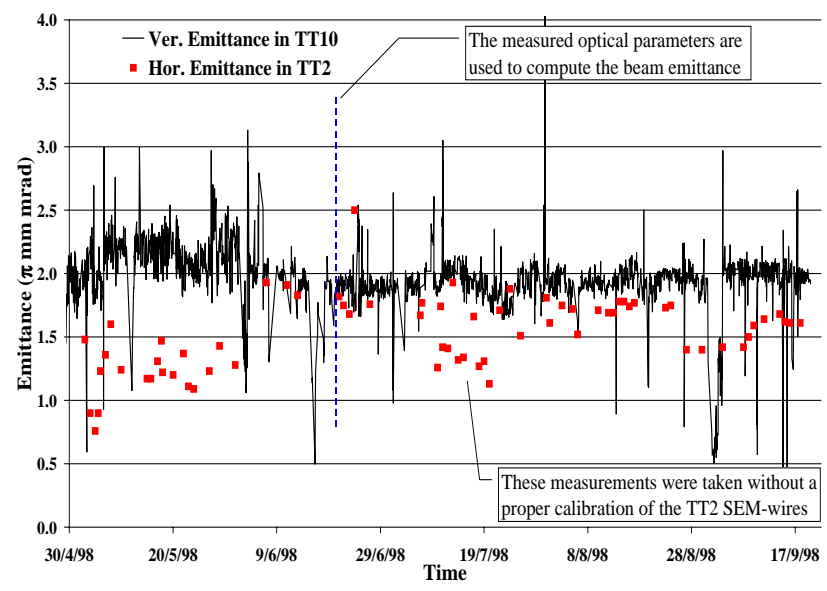

Figure 3: Comparison between the emittance value measured using monitors in TT2 and in TT10 during the 1998 run at $14 \mathrm{GeV} / \mathrm{c}$. The good trend due to the use of the measured optical parameters is visible

\subsection{The lead ion beam}

As a result of the betatron matching of the transfer line, the transmission through the SPS went up from $75 \%$ to $88 \%$ and the beam intensity delivered to the targets increased accordingly [9]. In Fig. 4 the average number of lead ions is shown: the sudden increase in the intensity is due to the new optics in TT2/TT10.

\section{CONCLUSIONS}

The study described in this paper will continue during the 1999 run with particular attention to: precise measurement of the beam energy and adjustment of its control value accordingly, extension of the error analysis to all measurement stages, measurement of the dependence of mismatch on extraction conditions (energy, extraction trajectory), online measurement of optical parameters and matching correction [10].

\section{ACKNOWLEDGEMENTS}

The support of the PS and SPS operation teams is acknowledged.

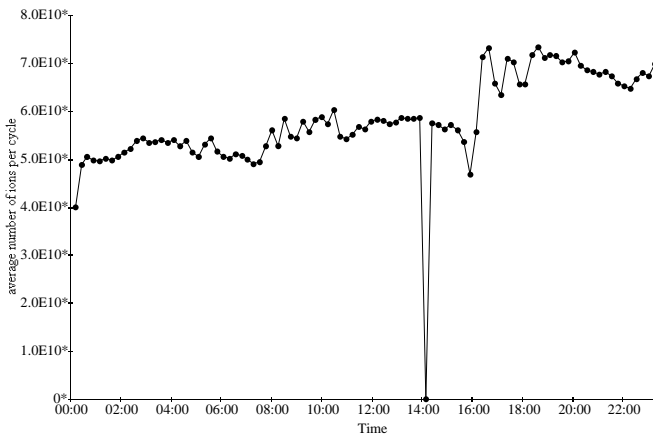

Figure 4: Average number of lead ions per cycle over 24 hours. The step function in efficiency is due to the matched optics in TT2/TT10.

\section{REFERENCES}

[1] P. Collier (ed.), "The SPS as Injector for LHC, Conceptual Design", CERN SL (DI) 97-07 (1997).

[2] G. Arduini, M. Giovannozzi, K. Hanke, J.-Y. Hémery, M. Martini, "MAD and BeamOptics Description of the TT2/TT10 Transfer Line - Part I: Optics without Emittance Exchange Insertion", CERN PS Note (CA) 98-014 and CERN SL Note (OP) 98-040 (1998).

[3] G. Arduini, M. Giovannozzi, K. Hanke, J.-Y. Hémery, "Study of the TT2/TT10 Transfer Line Optics via Transfer Matrix Measurement", CERN PS Note (CA) 98-020 and CERN SL-MD Note 98-058 (1998).

[4] G. Arduini, M. Giovannozzi, D. Manglunki, M. Martini, "Measurement of the optical parameters of a transfer line using multi-profile analysis", CERN PS (CA) 98-032 (1998).

[5] P. J. Bryant, K. Johnsen, "The principles of circular accelerators and storage rings", (Cambridge University Press, Cambridge, 1993).

[6] D. A. Edwards, M. J. Syphers, "An introduction to the physics of high energy accelerators", (John Wiley \& Sons, New York, 1993).

[7] G. Arduini, G. Crockford, C. Despas, M. Giovannozzi, K. Hanke, D. Manglunki, M. Martini, G. Métral, C. Niquille, "Betatron and dispersion matching of the TT2/TT10 transfer line for the $26 \mathrm{GeV} / \mathrm{c}$ fast extraction", CERN PS Note (CA) 99-07 and CERN SL Note 99-003 MD (1999).

[8] G. Arduini, G. Crockford, M. Giovannozzi, K. Hanke, D. Manglunki, M. Martini, G. Métral, C. Niquille, "TT2/TT10 transfer line studies for the $14 \mathrm{GeV} / \mathrm{c}$ continuous transfer", CERN PS Note (CA) 99-08 and CERN SL Note 99-013 MD (1999).

[9] G. Arduini, G. Crockford, C. Despas, M. Giovannozzi, K. Hanke, D. Manglunki, M. Martini, G. Métral, C. Niquille, "Betatron and dispersion matching of the TT2/TT10 transfer line for the fixed target lead ion beam", CERN PS Note (CA) 99-09 and CERN SL Note 99-018 MD (1999).

[10] G. Arduini, K. Hanke, "Tuning Knobs for the PS-SPS Transfer Line", these proceedings, (1999). 